\title{
ANÁLISE DA INFLUÊNCIA DA DORSIFLEXÃO NO EQUILÍBRIO POSTURAL EM MULHERESIDOSAS
}

\section{ANALYSIS OF THE DORSIFLEXION INFLUENCE ON THE POSTURAL BALANCE OF OLDER WOMEN}

Guilherme Henrique Benassi (ORCID: 0000-0002-9264-772X) ${ }^{1}$

Isadora Irmer Alves (ORCID: 0000-0003-0417-3999) ${ }^{2}$

Giovana Ponce de Miranda (ORCID: 0000-0001-7298-1205) ${ }^{2}$

Alex Silva Ribeiro (ORCID: 0000-0002-0312-8857)

Luiz Otávio Davanso (ORCID: 0000-0002-2413-6418) ${ }^{1}$

Edgar Ramos Vieira (ORCID: 0000-0002-1011-5077) 3

Márcio Rogério de Oliveira (ORCID: 0000-0002-8315-5117)

Autor correspondente:

Márcio Rogério de Oliveira

E-mail: marxroge@hotmail.com

\section{RESUMO}

O equilíbrio postural sofre alterações fisiológicas devido à idade, diminuindo a capacidade de se equilibrar na posição ortostática e/ou em um movimento dinâmico. O objetivo do presente estudo foi verificar se a dorsiflexão tem influência e se correlaciona com diferentes avaliações de equilíbrio postural em mulheres idosas. Um total de 54 mulheres idosas foram divididas em dois grupos: 1) grupo com baixa mobilidade de dorsiflexão (n=27, 68 anos); e 2) grupo com mobilidade de dorsiflexão normal ( $\mathrm{n}=27,68$ anos). Todas realizaram um teste de dorsiflexão para avaliação da mobilidade (teste de Lunge) e testes para avaliar o equilíbrio postural: teste de alcance funcional e postura bipodal com olhos abertos e fechados sobre uma plataforma de força. Os resultados encontrados demonstraram diferenças significativas entre os grupos para as condições alcance funcional e bipodal olho aberto $(\mathrm{p}<0,04)$. Para a relação entre as variáveis, a correlação encontrada foi entre a dorsiflexão e o teste de alcance funcional $(r=0,51 ; p<0,001)$. Os achados do presente estudo sugerem que a mobilidade reduzida do tornozelo pode influenciar no aumento da oscilação postural e que uma correlação moderada é encontrada entre a dorsiflexão e o alcance funcional em mulheres idosas fisicamente independentes.

Palavras-chave: Envelhecimento; Idoso; Equilíbrio Postural; Limitação da Mobilidade.
${ }^{1}$ Programa de Pós-Graduação em Exercício Físico na Promoção da Saúde. Universidade Unopar.

${ }^{2}$ Curso de Fisioterapia. Universidade Pitágoras Unopar.

${ }^{3}$ Departamento de Fisioterapia. Florida International University.

\begin{abstract}
Postural balance undergoes physiological changes because of the aging process, decreasing the ability to balance in an orthostatic position and / or in a dynamic movement. The aim of this study was to verify whether dorsiflexion has an influence and correlates with different assessments of postural balance in older women. A total of 54 older women were divided into two groups: 1) group with low dorsiflexion mobility ( $\mathrm{n}=27,68$ years) and 2) group with normal dorsiflexion mobility ( $\mathrm{n}=27,68$ years), all of them performed a dorsiflexion test to assess mobility (Lunge test) and tests to assess postural balance: functional reach test and bipodal posture with eyes open and closed on a force platform. The results found showed significant differences between the groups for the functional range and bipodal open eye conditions $(p<0.04)$. For the relationship between the variables, the correlation found was between dorsiflexion and functional reach test $(\mathrm{r}=0.51 ; \mathrm{p}=<0.001)$. The findings of the present study suggest that reduced mobility of the ankle may influence the increase in postural oscillation and a moderate correlation is found between dorsiflexion and functional reach in physically independent older women.
\end{abstract}

Keywords: Aging; Aged; Postural Balance; Mobility Limitation. 


\section{INTRODUÇÃO}

O equilíbrio postural é uma habilidade motora complexa que sofre alterações fisiológicas por causa da idade, afetando componentes periféricos e centrais, ocasionando prejuízos importantes na capacidade de se equilibrar, seja na posição ortostática e/ou em um movimento dinâmico ${ }^{1,2}$.

Um movimento articular de tornozelo reduzido demonstra ter um impacto negativo na função de membros inferiores e da marcha ${ }^{3}$. Nesse aspecto, cabe ressaltar que, na tentativa de restabelecer o equilíbrio postural, o corpo pode utilizar diferentes estratégias, e uma delas é a estratégia do tornozelo ${ }^{1}$. Tal método acontece em resposta do corpo diante das oscilações de baixa amplitude que se iniciam no tornozelo ${ }^{1}$. A capacidade de manter o equilíbrio postural é fundamental para diferentes atividades e pode ser exigida dependendo da dificuldade da tarefa e do ambiente ${ }^{4}$.

Evidências mostram que a menor amplitude de dorsiflexão pode influenciar significativamente o equilíbrio postural, e esse fator parece ser preditivo de quedas ${ }^{5,6}$ Todavia, esses aspectos são apresentados em testes de marcha e testes funcionais em adultos e idosos. Portanto, avaliar o movimento articular do tornozelo e identificar seu impacto no equilíbrio postural, principalmente, com medidas diretas e quantitativas denota ser importante para os idosos.

Existem diferentes meios para avaliar o movimento de dorsiflexão. Nesse sentido, o goniômetro é classificado como um método convencional ${ }^{7}$, mas que pode apresentar problemas de confiabilidade ${ }^{7-9}$. Por outro lado, aplicativos de celular têm sido utilizados como ferramenta de avaliação. Em um estudo recente ${ }^{10}$, essa ferramenta mostrou-se confiável para determinar a dorsiflexão da articulação do tornozelo.

Para avaliação do equilíbrio postural, medidas oriundas da plataforma de força são utilizadas, tendo como parâmetro a Oscilação do Centro de Pressão (COP), que são sinais válidos e confiáveis de comprometimento do equilíbrio postural entre os idosos ${ }^{11,12}$. Além disso, testes de campo auxiliam no rastreio de déficits de equilíbrio postural, e o teste de alcance funcional é utilizado para analisar os limites de estabilidade, que é essencial para a realização de atividades da vida diária com segurança, especialmente aqueles que envolvem alcançar um alvo ou objeto em direções diferentes ${ }^{13}$.

Avaliar o equilíbrio postural e as variáveis que podem influenciar essa capacidade é essencial para um diagnóstico clínico mais detalhado no campo da gerontologia e reabilitação ${ }^{14}$. Os estudos apontam que o sexo é um fator relacionado com as ocorrências de dependência funcional, sendo que as chances de as mulheres sofrerem quedas é duas vezes maior quando comparadas aos homens $s^{2,14,15}$. Nesse sentido, é importante determinar diferentes mecanismos que podem levar a um prejuízo no equilíbrio postural das mulheres. Com isso, até o momento, poucos estudos compararam o déficit de dorsiflexão com o equilíbrio postural em mulheres idosas. Dessa forma, o objetivo do estudo foi verificar se a dorsiflexão tem influência e se correlaciona com diferentes avaliações de equilíbrio postural em mulheres idosas.

\section{MÉTODOS}

\section{Participantes}

Trata-se de um estudo transversal, com participantes do sexo feminino recrutadas de forma voluntária e por conveniência da comunidade local por meio de contatos pessoais. Um total de 54 mulheres idosas completaram os testes e foram incluídas na análise. Os critérios de elegibilidade do presente estudo foram: mulheres com idade superior a 60 anos consideradas fisicamente independentes, 
classificado no nível 3 ou 4 da escala de Status Funcional proposto por Spirduso ${ }^{16}$; ausência de qualquer tipo de doença neurológica, metabólica e/ou ortopédica de alta gravidade, como cirurgia ou fratura em membros inferiores, ou, ainda, comprometimento no complexo articular do tornozelo (relato de entorse nos últimos 6 meses). Após a inclusão no estudo, as participantes foram divididas em dois grupos: Grupo 1) Grupo com baixa dorsiflexão (G1); e Grupo 2) Grupo com dorsiflexão normal (G2). Foram consideradas de baixa dorsiflexão aquelas participantes que apresentaram amplitude de movimento menor que $30^{\circ}$ no teste de Lunge ${ }^{17}$. A pesquisa foi aprovada pelo Comitê de Ética em Pesquisa da Universidade Pitágoras Unopar (parecer: 3.509.003).

\section{Procedimentos}

O presente estudo foi realizado no período de maio a julho de 2019. Todas as coletas de dados foram realizadas no Laboratório de Avaliação Funcional e Performance motora humana (Lafup) da Unopar, que possui iluminação e temperatura $\left( \pm 22^{\circ} \mathrm{C}\right)$ adequada para realização dos testes. As sessões de avaliação no laboratório foram de, no máximo, uma hora para cada participante. Todas as participantes foram familiarizadas com o equipamento e com os procedimentos antes de cada teste.

\section{Teste de Lunge}

As voluntárias foram instruídas previamente para se posicionarem na posição ortostática, descalças, com um pé à frente do outro, posição de tandem, e foram orientadas para que não levantassem o calcanhar do solo. Uma distância inicial entre o pé e a parede de $10 \mathrm{~cm}$ foi utilizada e determinada por meio de uma fita métrica. O membro contralateral foi posicionado atrás do membro de teste em uma posição confortável, e as mãos apoiadas na parede ajudavam a manter a estabilidade (figura 1). As participantes deveriam então alcançar a maior distância possível, levando o joelho à frente e em direção à parede de forma ativa. Um aplicativo de celular
(Compass, Iphone 7) foi posicionado 15 $\mathrm{cm}$ abaixo da tuberosidade anterior da tíbia para identificar a medida de dorsiflexão. Foram realizados três testes com a perna direita e três testes com a perna esquerda, sempre com o cuidado para que a voluntaria ficasse com os pés totalmente no solo, além de evitar movimentos compensatórios no seguimento do quadril e tronco. A posição máxima era o ponto em que o calcanhar começava a levantar do chão ou que o joelho tocasse na parede ${ }^{18,19}$; a maior amplitude (graus) alcançada foi utilizada nas análises.

Figura 1. Demonstração do teste de Lunge

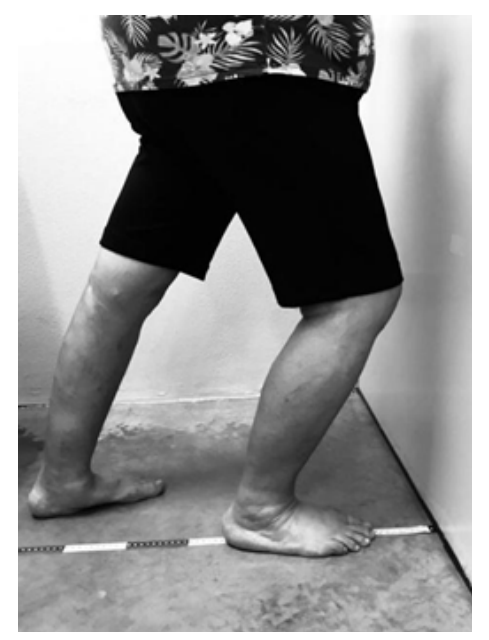

\section{Alcance funcional (alcance anterior)}

Nesse teste, a participante permanece em pé com os pés afastados na largura dos ombros e com o ombro flexionado de forma a manter os braços a $90^{\circ}$ do corpo. Uma fita métrica foi posicionada em um suporte na mesma altura do acrômio de cada avaliada (todas as participantes foram avaliadas elevando o membro superior direito). Sem movimentar os pés, foi solicitado à participante que alcançasse o mais longe possível à frente (anteriormente) sem perder o equilíbrio. O teste de alcance funcional foi criado por Duncan para medir os limites de estabilidade dos indivíduos, estendendo a mão para a frente em pé, e são definidos como a distância máxima que o centro de massa pode ser movido com segurança sem mudanças na base de apoio ${ }^{20}$. O teste de alcance funcional é rápido e fácil de aplicar, uma vez que requer poucos materiais ${ }^{20}$. Cada 
participante realizou o teste três tentativas, e a melhor medida foi retida para análise.

\section{Equilíbrio Postural}

Todas as participantes foram avaliadas sobre a plataforma de força BIOMEC400 (EMG System do Brasil, SP Ltda.), a qual possui quatro células de carga em posição retangular - ela mede $500 \times 500 \times 100 \mathrm{~mm}$ e pesa $22 \mathrm{~kg}$. A sensibilidade de cada sensor de carga é certificada por $0,015 \%$ para uma carga máxima de $1000 \mathrm{~N}$. A variação de $9.999 \mathrm{~N}$ da força aplicada para uma célula de carga corresponde a $120-\mathrm{mV}$ de variação de output, o qual tem um range que varia de 0 a $5 \mathrm{~V}$. O sistema usa um 16-bit analógico-digital conversor e filtros de rejeição de $50 \mathrm{~Hz}$. A força vertical de reação do solo é derivada de uma amostragem de $100 \mathrm{~Hz}$ para coleta de dados. $\mathrm{O}$ dado digital é transferido via cabo USB universal para um computador. Todos os sinais de força registrados pela plataforma são filtrados com filtro de banda passante baixo de $35-\mathrm{Hz}$ e de segunda ordem (Butterworth filter) para eliminar os ruídos elétricos ${ }^{21}$

Para aquisição e tratamento dos parâmetros de equilíbrio, foi utilizado o próprio software Bioanalysis da plataforma BIOMEC400, compilado com rotinas de computação de análises MATLAB (The Mathworks, Natick, MA). Os principais parâmetros de equilíbrio baseados no COP foram computados: área elipse $(95 \%)$ do COP (A-COP em $\mathrm{cm} 2$ ) e a velocidade média (VEL em $\mathrm{cm} / \mathrm{s}$ ) de oscilações do COP nas direções do movimento: Ântero-Posterior (A/P) e Médio-Lateral (M/L) ${ }^{22}$.

Para o protocolo de equilíbrio, foram realizadas duas tentativas com olhos abertos e duas tentativas com olhos fechados (totalizando 4 testes), ambas as condições experimentais com apoio bipodal durante 30 segundos, com 30 segundos de repouso entre cada tentativa. $\mathrm{O}$ protocolo de equilíbrio foi realizado com os pés descalços, braços ao longo do corpo e olhos direcionados para um alvo (cruz) referente a um ponto fixo de 2,5 metros de distância em relação à plataforma ${ }^{21}$.

\section{Análise estatística}

Os dados são apresentados por meio da estatística descritiva com medidas de tendência central, média e desvio-padrão. A distribuição paramétrica dos dados foi verificada pelo teste de Shapiro Wilk. Testes para comparação entre os grupos (baixa amplitude de movimento vs amplitude normal) e as variáveis de equilíbrio postural foram aplicados conforme a distribuição paramétrica dos dados (Teste t não pareado). Correlações entre as variáveis foram realizadas para identificar a associação entre as variáveis de equilíbrio postural (plataforma de força e teste de alcance funcional) e a mobilidade de tornozelo (Lunge). A correlação entre as variáveis foi classificada como: 0,0 até 0,30 , correlação insignificante; 0,30 a 0,50 , correlação baixa; 0,50 até 0,70 , correlação moderada; 0,70 até 1 , correlação forte ${ }^{23}$. Foi utilizado o software SPSS 17.0, e a significância adotada no estudo foi de $5 \%$.

\section{RESULTADOS}

A tabela 1 apresenta as características das participantes. Em geral, foram encontradas diferenças significativas entre os grupos (Grupo 1 e Grupo 2) para as variáveis de peso (G1 vs $\mathrm{G} 2, \mathrm{p}=0,004)$ e Índice de Massa Corpórea (IMC) (G1 vs $\mathrm{G} 2, \mathrm{p}=0,005)$ e amplitude de movimento (G1: $27 \pm 3$ vs $G 2: 36 \pm 4$ graus, $p<0,001)$. Para as outras variáveis (idade e altura), nenhuma diferença significativa foi encontrada $(\mathrm{p}>0,05)$. 
Tabela 1. Características das participantes. Londrina, 2019

\begin{tabular}{lcccc}
\hline \multicolumn{1}{c}{ Variável } & Total $(\mathbf{n = 5 4 )}$ & Grupo 1 (n=27) & Grupo 2 (n=27) & $\boldsymbol{p}$ \\
\hline Idade (anos) & $68(6)$ & $68(5)$ & $68(6)$ & 0,875 \\
Peso $(\mathrm{kg})$ & $67(11)$ & $71(10)$ & $63(9)$ & 0,004 \\
Altura $(\mathrm{m})$ & $1,55(0,5)$ & $1,56(0,5)$ & $1,55(0,6)$ & 0,833 \\
IMC $\left(\mathrm{kg} / \mathrm{m}^{2}\right)$ & $27(4)$ & $29(4)$ & $26(4)$ & 0,005 \\
\hline
\end{tabular}

Os dados são apresentados em média e desvio-padrão (DP). Grupo 1 = grupo considerado com baixa dorsiflexão; Grupo 2 = grupo considerado com dorsiflexão normal. IMC: Índice de massa Corpórea.

Para a comparação dos grupos, no que se refere ao equilíbrio postural na plataforma de força, somente a condição bipodal apresentou diferença significativa (G1 vs G2, p=0,030, (figura 2)). Nas demais comparações, não foram encontradas diferenças importantes. Por outro lado, no teste de alcance funcional, o aumento da amplitude de dorsiflexão resultou em melhor desempenho (G2: $32 \pm 5 \mathrm{~cm})$ quando comparado com baixa amplitude (G1: $26 \pm 7 \mathrm{~cm}$ ), com diferença significativa $\mathrm{p}=0,002$ entre os grupos (figura 3 ).

Figura 2. Comparação entre dorsiflexão e a área do centro de pressão para condição bipodal olhos abertos

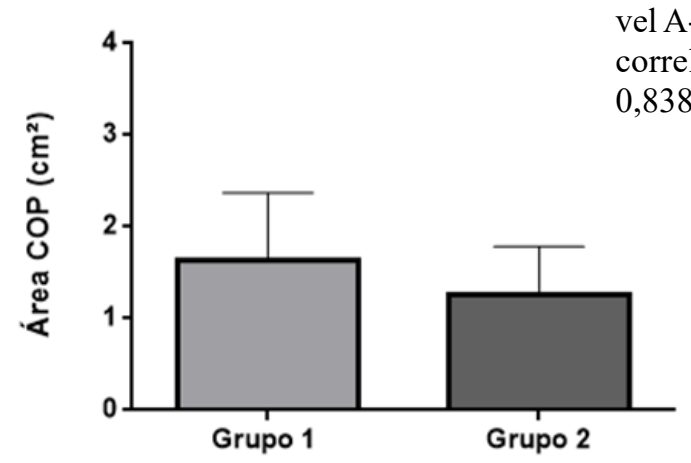

Figura 3. Comparação entre dorsiflexão e o teste de alcance funcional

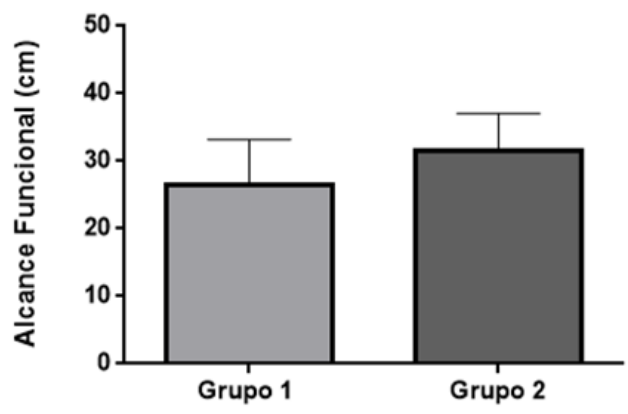

Em relação às associações entre as variáveis, foram encontradas correlações classificadas de fraca a moderada $(\mathrm{r}=-0,03$ / 0,51). Nesse sentido, a melhor correlação encontrada foi entre dorsiflexão e alcance funcional $(\mathrm{r}=0,51 ; \mathrm{p}<0,001)$. Para a variável A-COP na condição bipodal, obteve-se correlação considerada fraca $(r=-0,03 ; p$ $0,838)$. 
DISCUSSÃO

O presente estudo investigou a influência da dorsiflexão sobre o equilíbrio postural avaliado na plataforma de força e no teste de alcance funcional, bem como as correlações entre esses testes em mulheres idosas. Os principais achados apontam que a baixa mobilidade de dorsiflexão pode influenciar no equilíbrio postural, provocando instabilidade tanto em situações semiestáticas durante o apoio bipodal quanto em situações dinâmicas durante o alcance funcional. Além disso, uma correlação moderada e positiva foi encontrada entre dorsifflexão e alcance funcional, o que indica a associação entre maior mobilidade de dorsiflexão com maior alcance funcional.

Diferentes variáveis, como mobilidade, força muscular da extremidade inferior e postura do pé, podem influenciar no equilíbrio postural de idosos ${ }^{24,25}$. No entanto, este estudo se propôs a demonstrar como a mobilidade de dorsiflexão pode refletir no equilíbrio postural. No que se refere à comparação de baixa mobilidade e mobilidade normal, a comparação direta dessas variáveis para idosos ainda é limitada na literatura. No entanto, um recente estudo de revisão sistemática descreveu que idosos com histórico de quedas apresentam menor amplitude de movimento de dorsiflexão (teste de Lunge) em comparação com idosos sem histórico ${ }^{26}$. Por outro lado, evidências descrevem que a baixa mobilidade de dorsiflexão promove prejuízos para o equilíbrio postural e para a marcha porque a base de apoio para distribuição do centro de massa sofre alterações e diminui ${ }^{25,27}$.

Testes de equilíbrio na plataforma de força em posição semiestática (bipodal) parecem não ter correlação com a amplitude de dorsiflexão quando avaliados em cadeia cinética fechada (teste de Lunge) utilizando um aplicativo de celular. Todavia, para testes dinâmicos, como o alcance funcional realizado neste estudo, indicam associação com o equilíbrio postural. No estudo de Norris et al. ${ }^{24}$, foi descrito que a dorsiflexão e o equilíbrio postural de idosos apresentaram correlação de fraca a moderada entre as variáveis $(\mathrm{r}=$ $-0,03 / 0,53)$. Além disso, Menz et al. ${ }^{28}$ demonstraram que a mobilidade do tornozelo estava moderadamente correlacionada $(\mathrm{r}=0,51)$ com a oscilação postural. Embora alguns resultados de associação possam ser parecidos, vale ressaltar que as medidas de avaliação para o equilíbrio postural (limites de estabilidade $x$ medidor de oscilação) e alguns testes (teste de passo alternado $\mathrm{x}$ sentar e levantar) utilizados nos estudos diferem dos descritos neste manuscrito.

As participantes do estudo apresentam diferenças em algumas características iniciais, e cabe aqui destacar alguns pontos. Primeiro, no que se refere à diferença em relação ao peso, poucos estudos têm abordado esse tema em idosos, principalmente, mulheres. No entanto, ressaltamos que o peso corporal baixo ou alto pode projetar a força de gravidade causando maiores ou menores acelerações ao redor dos músculos do tornozelo; e, portanto, afetariam as medidas do COP aumentando ou diminuindo as oscilações a partir do torque do tornozelo ${ }^{29,30}$. Segundo, em relação ao IMC, existe evidência apontando que essa variável não influenciou no equilíbrio postural de idosos na plataforma de força. Pereira et al. ${ }^{31}$ dividiram 257 participantes em 4 grupos - baixo peso, peso normal, pré-obesidade e obesidade - e concluíram que o IMC e a massa gorda parecem não influenciar o equilíbrio dos idosos durante a postura unipodal. Por fim, cabe ressaltar que os participantes (homens) e as condições (baixo peso, peso normal, pré-obesidade e obesidade; unipodal) são diferentes da abordagem usada neste estudo; e, portanto, os resultados levantados aqui devem ser levados com cautela devido a esses fatores.

Os autores destacam que o ponto forte e a aplicação clínica deste estudo constam em direcionar a visão dos profissionais que trabalham com intervenção para um segmento que muitas vezes é esquecido durante os programas de exercício, que é a mobilidade de tornozelo. Estudos têm apontado ferramentas simples e acessíveis para avaliação desse segmento com importância clínica, para diferentes atividades funcionais ${ }^{32-34}$. Contudo, enfatizamos que os fatores que regulam equilíbrio são multifacetados (isto é, a integração de diferentes sistemas) e que isso poderia explicar a fraca correlação entre algumas medidas. Qualquer comprometimento no aspecto cognitivo, proprioceptivo (sensorial e motora), na força muscular ou coordenação motora pode resultar em déficits 
no equilíbrio postural em idosos, principalmente quando avaliados pela plataforma de força.

Por fim, este estudo apresenta algumas limitações que devem ser levadas em consideração: não ter realizado estudo de confiabilidade do teste com uma amostra prévia (apesar de que outros estudos já tenham demonstrado boa confiabilidade do teste de dorsiflexão); não ter avaliado o nível de atividade física das participantes, embora elas se considerassem sedentárias; e não ter incluído homens idosos. Portanto, os resultados aqui apresentados não devem ser aplicados para a população geral. Dessa forma, ressaltamos que outros estudos devem ser realizados com população, testes e materiais de avaliação de dorsiflexão diferentes, assim como outros testes na plataforma de força.

\section{CONCLUSÕES}

Os resultados deste estudo sugerem que a mobilidade reduzida do tornozelo pode influenciar no aumento da oscilação postural e que uma correlação moderada é encontrada entre a dorsiflexão e o alcance funcional em mulheres idosas fisicamente independentes. Esses achados indicam que avaliar a dorsiflexão pode ser importante para detectar déficits no equilíbrio postural de mulheres idosas.

\section{AGRADECIMENTOS}

À Fundação Nacional de Desenvolvimento do Ensino Superior Particular - Funadesp pelo apoio e incentivo à pesquisa.

\section{REFERÊNCIAS}

1. Horak FB. Postural orientation and equilibrium: What do we need to know about neural control of balance to prevent falls? Age Ageing. 2006;35(suppl_2):ii7-ii11.

2. Nakagawa HB, Ferraresi JR, Prata MG, Scheicher ME. Postural balance and functional independence of elderly people according to gender and age: Cross-sectional study. Sao Paulo Med J. 2017;135(3):260-265.

3. Burns J, Crosbie J. Weight bearing ankle dorsiflexion range of motion in idiopathic pes cavus compared to normal and pes planus feet. The Foot. 2005;15(2):91-94.

4. Peterka R. Sensorimotor integration in human postural control. J Neurophysiol. 2002;88(3):1097-1118.

5. Kemoun G, Thoumie P, Boisson D, Guieu JD. Ankle dorsiflexion delay can predict falls in the elderly. J Rehabil Med. 2002;34(6):278-283.

6. Hoch MC, Staton GS, McKeon PO. Dorsiflexion range of motion significantly influences dynamic balance. J Sci Med Sport. 2011;14(1):90-92.

7. Lundgren $P$, Nester C, Liu A, Arndt A, Jones R, Stacoff A, et al. Invasive in vivo measurement of rear-, mid-and forefoot motion during walking. Gait Posture. 2008;28(1):93-100.

8. Meyer DC, Werner CM, Wyss T, Vienne P. A mechanical equinometer to measure the range of motion of the ankle joint: Interobserver and intraobserver reliability. Foot Ankle Int. 2006;27(3):202-205.

9. Gatt A, Chockalingam N. Clinical assessment of ankle joint dorsiflexion: A review of measurement techniques. J Am Podiatr Med Assoc. 2011;101(1):59-69.

10. Banwell HA, Uden H, Marshall N, Altmann C, Williams CM. The iPhone measure app level function as a measuring device for the weight bearing lunge test in adults: A reliability study. J. Foot Ankle Res. 2019;12(1):37. 
11. Bauer C, Gröger I, Rupprecht R, Gaßmann KG. Intrasession reliability of force platform parameters in community-dwelling older adults. Arch Phys Med Rehabil. 2008;89(10):1977-1982.

12. Pajala S, Era P, Koskenvuo M, Kaprio J, Törmäkangas T, Rantanen T. Force platform balance measures as predictors of indoor and outdoor falls in community-dwelling women aged 63-76 years. J Gerontol A Biol Sci Med Sci. 2008;63(2):171-178.

13. Pickerill ML, Harter RA. Validity and reliability of limits-of-stability testing: a comparison of 2 postural stability evaluation devices. J Athl Train. 2011;46(6):600-606.

14. Assis VG, Marta SN, De Conti MHS, Gatti MAN, Simeão SFAP, De Vitta A. Prevalência e fatores associados à capacidade funcional de idosos na estratégia saúde da família em montes claros, minas gerais, brasil. Rev Bras Geriat Geront. 2014;17(1):153-163.

15. Nogueira SL, Ribeiro RC, Rosado LE, Franceschini SC, Ribeiro AQ, Pereira ET. Fatores determinantes da capacidade funcional em idosos longevos. Braz J Phys Ther. 2010;14(4):322-329.

16. Spirduso WW, Francis KL, MacRae PG. Physical dimensions of aging. Champaign, IL: Human Kinetics; 1995.

17. Baumbach SF, Braunstein M, Seeliger F, Borgmann L, Böcker W, Polzer H. Ankle dorsiflexion: What is normal? development of a decision pathway for diagnosing impaired ankle dorsiflexion and M. gastrocnemius tightness. Arch Orthop Trauma Surg. 2016;136(9):1203-1211.

18. Hall EA, Docherty CL. Validity of clinical outcome measures to evaluate ankle range of motion during the weight-bearing lunge test. J Sci Med Sport. 2017;20(7): 618-621.

19. Powden CJ, Hoch JM, Hoch MC. Reliability and minimal detectable change of the weight-bearing lunge test: A systematic review. Man Ther. 2015;20(4):524-532.

20. Duncan PW, Weiner DK, Chandler
J, Studenski S. Functional reach: A new clinical measure of balance. J Gerontol. 1990;45(6):M192-M197.

21. Da Silva RA, Bilodeau M, Parreira RB, Teixeira DC, Amorim CF. Age-related differences in time-limit performance and force platform-based balance measures during one-leg stance. J Electromyogr Kinesiol. 2013;23(3):634-639.

22. Oliveira MR, Silva RA, Dascal JB, Teixeira DC. Effect of different types of exercise on postural balance in elderly women: A randomized controlled trial. Arch Gerontol Geriatr. 2014;59(3):506-514.

23. Mukaka MM. A guide to appropriate use of correlation coefficient in medical research. Malawi Med J. 2012;24(3):69-71.

24. Norris E, Hubbuch E, Ford A, Allen W. The relationship of weight-bearing and non-weight bearing ankle dorsiflexion to balance and gait performance in young and older adults. Phys Ther Rehabil. 2016;3(6):1-8.

25. Horak FB, Henry SM, Shumway-Cook A. Postural perturbations: New insights for treatment of balance disorders. Phys Ther. 1997;77(5):517-533.

26. Menz HB, Auhl M, Spink MJ. Foot problems as a risk factor for falls in community-dwelling older people: A systematic review and meta-analysis. Maturitas. 2018;118:7-14.

27. Spink MJ, Fotoohabadi MR, Wee E, Hill KD, Lord SR, Menz HB. Foot and ankle strength, range of motion, posture, and deformity are associated with balance and functional ability in older adults. Arch Phys Med Rehabil. 2011;92(1):68-75.

28. Menz HB, Morris ME, Lord SR. Foot and ankle characteristics associated with impaired balance and functional ability in older people. J Gerontol A Biol Sci Med Sci. 2005;60(12):1546-1552.

29. Gray DS, Bray GA, Gemayel N, Kaplan K. Effect of obesity on bioelectrical impedance. Am J Clin Nutr. 1989;50(2):255-260.

30. Teasdale N, Hue O, Marcotte J, 
Berrigan F, Simoneau M, Doré J, et al. Benassi et al Reducing weight increases postural stability in obese and morbid obese men. Int $\mathrm{J}$ Obes. 2007;31(1):153-160.

31. Pereira C, Silva RA, Oliveira MR, Souza RD, Borges RJ, Vieira ER. Effect of body mass index and fat mass on balance force platform measurements during a one-legged stance in older adults. Aging Clin Exp Res. 2018;30(5):441-447.

32. Bennell K, Talbot R, Wajswelner H, Techovanich W, Kelly D, Hall A. Intra-rater and inter-rater reliability of a weight-bearing lunge measure of ankle dorsiflexion. Aust J Physiother. 1998;44(3):175-180.

33. Hoch MC, Staton GS, McKeon JMM, Mattacola CG, McKeon PO. Dorsiflexion and dynamic postural control deficits are present in those with chronic ankle instability. J Sci Med Sport. 2012;15(6):574-579.

34. Menz HB, Tiedemann A, Kwan MM, Latt MD, Sherrington C, Lord SR. Reliability of clinical tests of foot and ankle characteristics in older people. J Am Podiatr Med Assoc. 2003;93(5):380-387.

Recebido: 27/04/2020 